Pacific Journal of Mathematics

ACTIONS OF FUNCTIONS IN BANACH ALGEBRAS 


\title{
ACTIONS OF FUNCTIONS IN BANACH ALGEBRAS
}

\author{
Frances F. Gulick
}

This paper introduces the concept of a function $f$ (defined on the open unit disk $U$ of the complex plane) acting in a Banach algebra $\mathfrak{A}$. In general, $f$ acts in $\mathscr{U}$ if there exists a mapping $x \rightarrow f(x)$ from $\{x \in \mathfrak{X}: \sigma(x) \subset U\}=\mathfrak{U}_{1}$ into $\mathfrak{U}$ such that for every maximal commutative subalgebra $\mathscr{C}$ of $\mathfrak{U},\{f(x): x \in$ $\left.\mathfrak{A}_{1} \cap \mathscr{C}\right\}$ is contained in $\mathscr{C}$ and $(f(x))^{\wedge}=f \circ \hat{x}\left(x \in \mathscr{C} \cap \mathfrak{X}_{1}\right)$ on the maximal ideal space of $\mathscr{C}$. After some properties of actions in general Banach algebras are established, attention is restricted to a subalgebra $\mathfrak{U}$ of the algebra $C_{p}$ of compact operators on a Hilbert space such that $\mathfrak{A}$ contains a normal operator of infinite rank. If $\mathfrak{U} \subset C_{\infty}$ and $\mathfrak{U}$ contains only normal operators, then a necessary and sufficient condition for $f$ to act in $\mathfrak{U}$ is that $f$ be continuous at zero and $f(0)=0$. For a more restricted class of subalgebras of $C_{p}, 1 \leqq p<\infty$, it is shown that $f$ defines an action in $\mathfrak{A}$ if, and only if, $f$ is Höldercontinuous at zero with $f(0)=0$.

Let $\mathfrak{A}$ be a commutative Banach algebra with identity $e$, let $\mathfrak{A}_{1}$ be the open unit ball in $\mathfrak{U}$ and let $x \rightarrow \widehat{x}$ be the Gelfand map. In addition, let $f$ be a function defined and analytic on the open unit disk $D$ of the complex numbers. Under these conditions a Banach algebraic analogue of the Cauchy integral formula appears which asserts that for each $x \in \mathfrak{A}_{1}$, there is an element $f(x) \in \mathfrak{A}$ satisfying the equation $f(x)=\int_{\gamma} f(\zeta)(\zeta e-x)^{-1} d \zeta$, where $\gamma$ denotes the boundary of $D$. This equation promptly yields the important functional equation

$$
(f(x))^{\wedge}=f \circ \hat{x},
$$

which verbally says that homomorphisms on $\mathfrak{A}$ commute with $f$. All this belongs to the basic theory of Banach algebras (see, for instance, page 203 of [10]).

What is important for us is that if we are given a commutative Banach algebra $\mathfrak{A}$ with identity $e$, and a function $f$ analytic on $D$, then we can define a natural map $x \rightarrow f(x)$ of $\mathfrak{A}_{1}$ into $\mathfrak{A}$ such that $(f(x))^{\wedge}=f \circ \hat{x}$. It is this result which forms the starting point for our paper. In order to transform the result into our final framework, let us tamper with the hypotheses we have assumed and then we will alter the conclusions so that they will make sense and be relevant.

The requirement that $\mathfrak{A}$ possess an identity can be dropped if the analytic function $f$ has the property that $f(0)=0$; then $f(x)$ is still in $\mathfrak{A}$ and the functional equation remains valid and in the process one 
need only alter the integral formula slightly (see p. 79 of [7]).

If $\mathfrak{A}$ is not necessarily commutative, then of course there need not exist complex-valued homomorphisms defined on the whole of 2 . Nevertheless, if we require that $f(x)$ be in a maximal commutative subalgebra $\mathscr{C}$ which contains $x$, and if the map $x \rightarrow \hat{x}$ denotes the Gelfand map on the commutative algebra $\mathscr{E}$ rather than on $\mathfrak{A}$, then for $f$ analytic on the unit disk $D$, the functional equation $(f(x))^{\wedge}=f \circ \hat{x}$ holds and the map $x \rightarrow f(x)$ of $\mathfrak{A}_{1}$ into $\mathfrak{A}$ has the required properties. Note that the Gelfand map in general depends upon the element $x$, a natural circumstance if $\mathfrak{A}$ is not commutative.

Finally, if we relax the condition that $f$ be analytic, then it is not clear that the integral formula need be defined at all, let alone yield an element of $\mathfrak{U}$. Indeed, we must ask if we can define the map $x \rightarrow f(x)$ satisfying the equation $(f(x))^{\wedge}=f \circ \widehat{x}$, and for what $f$ such a map exists. The question of existence is a difficult one and, without restriction, much too general. Only in concrete cases can one hope to conclude anything definitive about the existence of maps $x \rightarrow f(x)$ satisfying the functional equation. One such example already studied concerns the algebra of periodic functions with absolutely convergent Fouries series. Wiener [14] and Levy [6] began the study and Katznelson [5] completed it. Later the quartet consisting of Helson, Kahane, Katznelson and Rudin [4] studied the same question for the group algebra over a locally compact abelian group.

In order to facilitate our discussion, let us say that $f$ acts in $\mathfrak{A}$ (or $f$ defines an action in $\mathfrak{A}$ ) if there is a map $x \rightarrow f(x)$ from the open unit sphere $\mathfrak{A}_{1}$ of $\mathfrak{U}$ into $\mathfrak{X}$ such that for any maximal commutative subalgebra $\mathscr{C}$ of $\mathfrak{A}$ and $x \in \mathscr{C} \cap \mathfrak{A}_{1}, f(x) \in \mathscr{C}$ and $(f(x))^{\wedge}=f \circ \hat{x}$. This paper is devoted to analyzing properties of actions and studying those actions in algebras of operators on Hilbert space.

More explicitly, in $\S 3$ we show that if $\mathfrak{A}$ is semi-simple and commutative, then any action must necessarily be unique (Proposition 3.8). In addition, if $\mathfrak{A}$ contains no identity, then either $f(0)=0$ or else $\mathfrak{U}$ must contain a nonzero central idempotent (Proposition 3.6). Finally, the action of one function in $\mathfrak{A}$ gives rise to the notion of an algebra of functions defining an action in $\mathfrak{A}$, as we mention at the end of the section.

The remainder of the paper concerns itself with a study of actions in certain subalgebras of compact operators on a Hilbert space. Our theorems concern algebras which contain at least one normal operator of infinite rank.

In $\S 4$ we prove for the well-known class $C_{p}(1 \leqq p \leqq \infty)$ of compact operators on Hilbert space some elementary properties that we need in the sequel and we discuss actions on closed subalgebras of $C_{p}$. 
This leads us to $\S 5$, where we show that if 2 is a closed subalgebra of $C_{p}$ which contains a normal operator of infinite rank and if $f$ acts in $\mathfrak{A}$, then $f$ is continuous at 0 and $f(0)=0$ (Proposition 5.1). The converse to this theorem we prove for $p=\infty$ (Theorem 5.2). On the other hand, if $1 \leqq p<\infty$, then we can show that if $\mathfrak{U}$ is a closed subalgebra of $C_{p}$ which contains only normal operators, then a function defined on the open unit disk, Hölder-continuous at 0 with $f(0)=0$ does in fact define an action in $\mathfrak{A}$ (Proposition 5.3). Under additional hypotheses on $\mathfrak{A}$ we prove that Hölder-continuity of $f$ at 0 and $f(0)=0$ characterizes all actions in $\mathfrak{A}$ (Theorem 5.4). Thus we see that if $f(0)=0$, then continuity of $f$ at 0 is in general too weak to ensure that $f$ act in $\mathfrak{X}$, while Hölder-continuity at 0 is stronger than is usually needed to ensure that $f$ act in $\mathfrak{A}$.

Further results for functions acting in certain closed subalgebras of $C_{p}$ have been obtained. Some of these deal with the relationship between continuous functions and a limit property of their action in $\mathfrak{A}$. Since these were only by-products of the work to determine the class of functions which act in closed subalgebras of $C_{p}$, they were omitted for the sake of greater conciseness.

2. Preliminary discussion. Let $\mathfrak{A}$ be a Banach algebra over the complex numbers $C$. If $\mathfrak{U}$ has an identity $e$, then the spectrum of $x$ in $\mathfrak{A}$ is $\sigma(x)=\{\lambda: \lambda e-x$ has no inverse in $\mathfrak{A}\}$; if $\mathfrak{A}$ has no inverse, then the spectrum of $x$ in $\mathfrak{X}$ is $\sigma(x)=\{\lambda \neq 0: x / \lambda$ has no quasi-inverse in $\mathfrak{X}\}$ (see [11]). We let $\sigma_{0}(x)$ denote the nonzero elements of $\sigma(x)$. One theorem we shall refer to repeatedly is that for each decomposition of $\sigma(x)$ into nonempty, disjoint, relatively open and closed sets $\sigma_{1}, \sigma_{2}$ there exists an idempotent $e_{\sigma_{1}}(x)$ defined by the Cauchy integral formula such that the spectrum of $x e_{\sigma_{1}}(x)$ is precisely $\sigma_{1}$.

Throughout the paper the symbol $U$ denotes the open unit disk in the complex plane. For a given Banach algebra, we let $\mathfrak{A}_{1}$ be the set $\{x \in \mathfrak{V}: \sigma(x) \subset U\}$, or equivalently, $\mathfrak{X}_{1}$ is the open unit ball in the topology generated by the spectral radius seminorm.

We write the maximal ideal space of a commutative Banach algebra $\mathfrak{U}$ as $M_{\mathfrak{U}}$, and we let $x \rightarrow \hat{x}$ be the Gelfand map on $\mathfrak{X}$ defined by $\hat{x}(h)=h(x), h \in M_{\mathfrak{X}}$, for each $x \in \mathfrak{Y}$. We note that if $\mathfrak{U}$ has an identity, then the range of $\hat{x}$ is $\sigma(x)$, while if $\mathfrak{A}$ does not have an identity, then $\sigma(x)=\hat{x}\left(M_{\mathfrak{V}}\right) \cup\{0\}[10,11]$. Let $C_{0}\left(M_{\mathfrak{Q}}\right)$ be the space of all functions continuous on $M_{\mathfrak{A}}$ and vanishing at infinity.

Let $H$ be a Hilbert space with norm $\|\cdot\|$. Denote by $L(H)$ the Banach algebra of continuous linear operators $T: H \rightarrow H$ under the operator norm $\|T\|$. We let $C_{\infty}(H)$ be the norm-closed two-sided ideal of compact operators in $L(H)$. If we fix the $H$, then we usually will abbreviate $C_{\infty}(H)$ to $C_{\infty}$. The subalgebra $C_{0}$ of $C_{\infty}$ which consists of 
operators of finite rank is dense in the operator-norm in $C_{\infty}$.

3. Definition of action and properties of actions. We now present the definition basic to the entire paper.

Definition 3.1. If a function $f$ is defined on the unit disk, then $f$ acts in the Banach algebra $\mathfrak{A}$ if there exists a map $x \rightarrow f(x)$ of $\mathfrak{A}_{1}$ into $\mathfrak{A}$ such that whenever $\mathscr{C}$ is a maximal commutative subalgebra of $\mathfrak{A}$ and $x \in \mathscr{C} \cap \mathfrak{A}_{1}$, then $f(x) \in \mathscr{C}$ and $(f(x))^{\wedge}=f \circ \hat{x}$ on $M_{\mathscr{C}}$. The mapping $x \rightarrow f(x)\left(x \in \mathfrak{A}_{1}\right)$ is called an action of $f$ in $\mathfrak{A}$.

We depart from previous usage of " $f$ operates in $\mathfrak{X}$ " in order to have a name for the mapping $x \rightarrow f(x)$ and yet keep our terminology related.

Examples of actions are well-known as we see by the following examples.

EXAMPLE 3.2. Let $\mathfrak{A}$ be a Banach algebra with an identity $e$ and $f$ a function analytic on $U$. For each $x \in \mathfrak{A}_{1}$ define $f(x)$ by means of the Cauchy integral formula $f(x)=\frac{1}{2 \pi i} \int_{r} f(\zeta)(\zeta e-x)^{-1} d \zeta$, where $\gamma$ is a circle of radius $r$ with $\sigma(x)$ in its interior. As a result of the homomorphism theorem [10, p. 203] the mapping $x \rightarrow f(x)$ is an action of $f$ in $\mathfrak{A}$.

ExAmPLe 3.3. Let $\mathfrak{A}=L_{1}(G), G$ a locally compact abelian group, and let $\Gamma$ be the dual group. In this case Definition 3.1 reduces to the statement that $f$ acts in $\mathfrak{A}$ if for every $x \in \mathfrak{A}_{1}$, there exists $f(x)$ in $\mathfrak{A}$ such that $(f(x))^{\wedge}=f \circ \hat{x}$ on $\Gamma$. This is essentially the definition of a function operating in $L_{1}(G)$ given in [4], [5], [13]. It is shown in [4] and [13] that a function $f$ with domain the interval $(-1,1)$ operates in $L_{1}(G)$ if, and only if, $f$ is real-analytic and $f(0)=0$ when $G$ is not compact. Thus a function $f$ defined on the unit disk acts in $L_{1}(G)$ if, and only if, $f$ is analytic.

ExAmple 3.4. Let $T$ be a normal, bounded, compact linear operator defined on a Hilbert space and let $\mathfrak{A}$ be the $C^{*}$-algebra generated by $T$. Let $f$ be a function defined on the unit disk which is continuous at zero with $f(0)=0$. Since $\sigma_{0}(T)$ and $M_{\mathfrak{Q}}$ are homeomorphic with zero and the zero homomorphism corresponding [10, p. 202] we can regard $f$ as an element of $C_{0}\left(M_{\mathfrak{A}}\right)$. The algebra $C_{0}\left(M_{\mathfrak{Y}}\right)$ is isometricallyisomorphic to $\mathfrak{X}\left[10\right.$, p. 230] and hence for every $S \in \mathfrak{A}_{1}$ there exists $f(S) \in \mathfrak{A}$ such that $(f(S))^{\wedge}=f \circ \hat{S}$ on $M_{\mathfrak{Q}} \cdot \quad$ (For a characterization of functions acting in $\mathfrak{A}$ see $\S 5$.) 
In the definition of $f$ acting in $\mathfrak{A}$ we require that $f$ be defined on the unit disk $U$. All theorems we prove hold if $U$ is an arbitrary complex domain with only minor modifications in the proofs.

The spectral mapping theorem [1, p. 569] shows that for the action of Example 3.2 the spectrum of $f(x)$ is the set $\{f(\lambda): \lambda \in \sigma(x)\}$. The following proposition shows that in general we have $\sigma(f(x))=\{f(\lambda): \lambda \epsilon$ $\sigma(x)\}$ if $\mathfrak{A}$ has an identity.

Proposition 3.5. Let $f$ be a function which acts in $\mathfrak{A}$. Then $f$ acts in any maximal commutative subalgebra of $\mathfrak{A}$. If $\mathfrak{A}$ has an identity, then for each $x \in \mathfrak{A}_{1}, \sigma(f(x))=\{f(\lambda): \lambda \in \sigma(x)\}$. If $\mathfrak{A}$ does not have an identity, $x \in \mathfrak{X}_{1}$ and $\mathscr{C}$ is a maximal commutative subalgebra of $\mathfrak{X}$ such that $x \in \mathscr{C}$, then $\sigma(f(x))=\left\{f(h(x)): h \in M_{\mathscr{E}}\right\} \cup\{0\}$.

Proof. The fact that $f$ acts in any maximal commutative subalgebra of $\mathfrak{A}$ follows immediately from Definition 3.1. If $x \in \mathfrak{A}_{1}$ and $\mathscr{C}$ is a maximal commutative subalgebra of $\mathfrak{A}$ such that $x \in \mathscr{C}$, then $f(x) \in \mathscr{C}$ and $\sigma_{\mathfrak{A}}(f(x))=\sigma_{\mathscr{C}}(f(x))=(f(x))^{\wedge}\left(M_{\mathscr{C}}\right) \quad(\cup\{0\}$ if $\mathfrak{A}$ does not have an identity), while $\sigma_{\mathfrak{X}}(x)=\hat{x}\left(M_{\mathscr{C}}\right)(\cup\{0\}$ if $\mathfrak{A}$ does not have an identity).

Proposition 3.6. Suppose $\mathfrak{A}$ does not contain an identity but does contain an element with nonzero spectrum. If $f$ acts in $\mathfrak{U}$, then either (i) $f(0)=0$ and the image of $0 \in \mathfrak{A}$ is in the radical of $\mathfrak{A}$ or (ii) $\mathfrak{A}$ contains a nonzero central idempotent.

Proof. Choose $x \in \mathfrak{A}$ such that $\sigma(x) \neq\{0\}$ and let $\mathscr{C}$ be a maximal commutative subalgebra of $\mathfrak{A}$ such that $x \in \mathscr{C}$. Since $0 \in \mathscr{C}, f(\underline{0})$ is in $\mathscr{C}$ and $\sigma(f(\underline{0}))=\{f(0), 0\}$ (Proposition 3.5). If $f(0) \neq 0$, then $f(0)$ is an isolated point of $\sigma(f(\underline{0}))$ so there exists an idempotent $e \in \mathfrak{A}$ which commutes with all elements of $\mathfrak{A}$ since $f(\underline{0})$ commutes with all elements of $2(10$, p. $203 ; 1$, p. 568]. On the other hand, if $f(0)=0$, then $\sigma(f(\underline{0}))=\{0\}$. To show that $f(\underline{0})$ is in the radical of $\mathfrak{A}$ we need to show that $f(\underline{0})+y f(\underline{0})$ has a quasi-inverse for all $y \in \mathfrak{A},[1, \mathrm{p} .55]$. This follows from the fact that $f(\underline{0})$ is in every maximal commutative subalgebra of $\mathfrak{X}$ and that in each such algebra its spectrum is $\{0\}$ $[11$, p. 112].

Suppose $y$ and $x$ commute and that $f(x)$ is defined. Then do $y$ and $f(x)$ also commute? If $\mathfrak{A}$ is a $*$-algebra and $x$ is normal in $\mathfrak{A}$, then is $f(x)$ normal? These questions are answered by the following proposition and its corollary.

Proposition 3.7. If $f$ acts in $\mathfrak{A}$ and $x \in \mathfrak{A}_{1}$ commutes with $y \in \mathfrak{A}$, 
then $f(x)$ and $y$ commute.

Proof. If $x \in \mathfrak{N}_{1}$ and $y$ commute, then there exists a maximal commutative subalgebra $\mathscr{C}$ of $\mathfrak{A}$ which contains $x$ and $y$. By definition of action $f(x) \in \mathscr{C}$ and hence $f(x)$ and $y$ commute.

COROLlary 3.7.1. If $\mathfrak{A}$ is a *algebra, $f$ acts in $\mathfrak{U}$ and $x \in \mathfrak{A}_{1}$ is normal, then $f(x)$ is normal.

Proof. As a result of Proposition $3.7 f(x)$ and $x^{*}$ commute. But then $(f(x))^{*} x=\left(x^{*} f(x)\right)^{*}=\left(f(x) x^{*}\right)^{*}=x(f(x))^{*}$. Since $(f(x))^{*}$ thus commutes with $x, f(x)$ and $f(x)^{*}$ commute (Proposition 3.7).

A natural question to ask about the action of a function in an algebra is whether a mapping satisfying Definition 3.1 need be unique. The answer is given in part in the following proposition (which is an immediate consequence of the definition of a function acting in $\mathfrak{U}$ ) and two examples.

Proposition 3.8. If $\mathfrak{A}$ is a semisimple, commutative Banach algebra, then a function $f$ can act in $\mathfrak{X}$ in at most one way.

To emphasize the importance of the hypothesis in Proposition 3.8 we present two examples which show that it is possible to define more than one action of a function in an algebra which is commutative but not semisimple or an algebra which is semisimple but not commutative.

EXAMPLE 3.9. Let $\mathfrak{A}$ be the algebra of $2 \times 2$ matrices of the form $\left(\begin{array}{ll}a & b \\ 0 & a\end{array}\right), a, b \in \boldsymbol{C}$. Then $\mathfrak{A}$ is a commutative algebra which is not semisimple (e.g., $\left(\begin{array}{ll}0 & 1 \\ 0 & 0\end{array}\right)$ has zero spectrum). Choose a differentiable function $f \in C(U)$ and a function $g: U \rightarrow C$. Regard $f$ to be a function of two real variables and set $f_{1}(z)=\partial f / \partial x$. Define the mapping $T \rightarrow$ $f_{g}(T)$ on $\mathfrak{U}_{1}$ by setting

$$
f_{g}(T)=f(a) I+f_{1}(a) g(a)(T-a I)=\left(\begin{array}{cc}
f(a) & f_{1}(a) g(a) b \\
0 & f(a)
\end{array}\right)
$$

for $T=\left(\begin{array}{ll}a & b \\ 0 & a\end{array}\right) \in \mathfrak{A}_{1}$. Clearly, $f_{g}(T) \in \mathfrak{A}$ and $\left(f_{g}(T)\right)^{\wedge}=f \circ \hat{T}$ on $M_{\mathfrak{X}}$.

EXAMPLE 3.10. Let $\mathfrak{A}$ be the full algebra of $2 \times 2$ matrices. Then $\mathfrak{A}$ is simple (and hence semisimple) but not commutative. Let $f \in C(U)$ 
be differentiable. For each function $g: U \rightarrow C$ define a map $T \rightarrow f_{g}(T)$ on $\mathfrak{N}_{1}$ as follows: if $\sigma(T)=\{\lambda\} \subset U$, set $f_{g}(T)=f(\lambda) I+f_{1}(\lambda) g(\lambda)(T-\lambda I)$, while if $\sigma(T)=\{\lambda, \mu\} \subset, \lambda \neq \mu$, set $f_{g}(T)=(\lambda-\mu)^{-1}[f(\lambda)(T-\mu I)-$ $f(\mu)(T-\lambda I)]$. For each $g: U \rightarrow C$ the mapping $T \rightarrow f_{g}(T), T \in \mathfrak{A}_{1}$, is an action of $f$ in $\mathfrak{U}$. Thus, in this example, as well as in Example 3.9 , we have defined many different actions of $f$ in $\mathfrak{A}$.

Suppose two functions $f$ and $g$ act in $\mathfrak{A}$ with actions $x \rightarrow f(x)$ and $x \rightarrow g(x), x \in \mathfrak{A}_{1}$, respectively. Then the mappings $x \rightarrow \alpha f(x)+g(x)$ $(\alpha \in C)$ and $x \rightarrow f(x) g(x)$ from $\mathfrak{X}_{1}$ into $\mathfrak{A}$ are actions of the functions $\alpha f+g$ and $f g$ (pointwise product), respectively, in $\mathfrak{A}$. Thus the set of all functions which act in $\mathfrak{A}$ is itself an algebra $\mathscr{M}$. Is there a map from $\mathscr{C} \times \mathfrak{U}_{1}$ into $\mathfrak{A}$ which is an algebra homomorphism on $\mathscr{C}$ when restricted to the set $\mathscr{M} \times\{x\}\left(x \in \mathfrak{H}_{1}\right)$ and an action of $f \in \mathscr{M}$ in $\mathfrak{U}$ when restricted to $\{f\} \times \mathfrak{A}_{1}$ ? These questions lead to a consideration of the problem of functions with finite range and the next proposition.

Proposition 3.11. Let $f$ be a function which acts in $\mathfrak{A}$. Let a be an isolated point of the range of $f$ with $a \neq 0$ if $\Re$ does not have an identity. Then the characteristic function $\chi_{a}$ of the set $f^{-1}(a)=$ $\{t \in U: f(t)=a\}$ acts in $\mathfrak{A}$ and the action is uniquely determined by the condition $\chi_{a}(x)^{2}=\chi_{a}(x)$.

Proof. If $\sigma(x) \subset U$ and $a \in \sigma(f(x))$, then $a$ is an isolated point (different from zero if $\mathfrak{A}$ does not have an identity). Hence there exists an idempotent $e_{a}(x) \in \mathfrak{A}$ such that if $\mathscr{C}$ is a maximal commutative subalgebra of $\mathfrak{U}$ with $x \in \mathscr{C}$ then $e_{a}(x) \in \mathscr{C}$ and $\left(e_{a}(x)\right)^{\wedge}$ is the characteristic function of the set $\left\{h \in M_{\mathscr{C}}: f(h(x))=a\right\}$. Thus $\left(e_{a}(x)\right)^{\wedge}=$ $\chi_{a} \circ \hat{x}$ on $M_{\mathscr{C}}$. (cf. $\left.\S 2\right)$. Define $\chi_{a}(x)\left(x \in \mathfrak{N}_{1}\right)$ by setting $\chi_{a}(x)=e_{a}(x)$ if $a \in \sigma(f(x))$ and $\chi_{a}(x)=0$ otherwise. This mapping $x \rightarrow \chi_{a}(x)\left(x \in \mathfrak{N}_{1}\right)$ is an action of $\chi_{a}$ in $\mathfrak{U}$ such that $\chi_{a}(x)^{2}=\chi_{a}(x)$ for all $x \in \mathfrak{A}_{1}$.

If $x \rightarrow e(x) \quad\left(x \in \mathfrak{X}_{1}\right)$ were another action of $\chi_{a}$ in $\mathfrak{A}$ such that $(e(x))^{2}=e(x)$ for all $x \in \mathfrak{A}_{1}$, then we would have $(e(x))^{\wedge}=\left(\chi_{a}(x)\right)^{\wedge}$ on $M_{\mathscr{G}}$ for any maximal commutative subalgebra $\mathscr{C}$ of $\mathfrak{A}$ such that $x \in \mathscr{C}$. Thus $e(x)-\chi_{a}(x) e(x)$ and $\chi_{a}(x)-\chi_{a}(x) e(x)$ are commuting idempotents with zero spectrum; hence $e(x)=\chi_{a}(x)$ for all $x \in \mathfrak{A}_{1}$ [9, p. 41].

COROLLARY 3.11.1. If a function $f$ with finite range $\left\{a_{1}, a_{2}, \cdots\right.$, $\left.a_{n}\right\}$ acts in $\mathfrak{A}$, then for each $x \in \mathfrak{A}_{1}$ there exist pairwise orthogonal $i d e m p o t e n t s e_{1}(x), e_{2}(x), \cdots, e_{n}(x)$ such that the mapping $x \rightarrow \sum_{i=1}^{n} a_{i} e_{i}(x)$, $x \in \mathfrak{U}_{1}$, is an action of $f$ in $\mathfrak{U}$ with the property that $\left(\sum_{i=1}^{n} a_{i} e_{i}(x)\right)^{k}=$ 
$\sum_{i=1}^{n} a_{i}^{k} e_{i}(x)$ for all $x=1,2, \cdots$.

Proof. For each $a_{i}, i=1,2, \cdots, n$, the characteristic function $\chi_{i}$ of the set $f^{-1}\left(a_{i}\right)$ defines an action $x \rightarrow e_{i}(x)$ in $\mathfrak{A}$ which is uniquely determined by the condition $\left(e_{i}(x)\right)^{2}=e_{i}(x)$. From the construction in the proof of Proposition 3.11 we see that $e_{i}(x) e_{j}(x)$ is zero if $i \neq j$. It then follows that the map $x \rightarrow \sum_{i=1}^{n} a_{i} e_{i}(x), x \in \mathfrak{A}_{1}$, is an action of $f$ in $\mathfrak{U}$ with the desired property.

If $f$ has finite range $\left\{a_{1}, a_{2}, \cdots, a_{n}\right\}$, then the characteristic function of each set $f^{-1}\left(a_{j}\right)$ is in the algebra of polynomials in $f$ and the action of this characteristic function is uniquely determined by the idempotency which is consistent with multiplication. Thus for a function $f$ which has finite range and acts in $\mathfrak{A}$, the action of Corollary 3.11.1 is the only one which is consistent with multiplication.

Now we return to the question of extending the action of a function $f_{0}$ to a mapping $\varphi$ from $\mathscr{C} \times \mathscr{N}_{1}$ into $\mathfrak{U}$ which is an algebra homomorphism on $\mathscr{C}$ when restricted to $\mathscr{C} \times\{x\}$. While we may not be able to obtain the mapping $\varphi$ for all of $\mathscr{C}$ we can obtain the desired type of mapping for subalgebra of $\mathscr{C}$.

Let $\mathscr{C}$ be the algebra of all functions which act in $\mathfrak{A}$. Choose $f_{0} \in \mathscr{C}$ and an action $x \rightarrow f_{0}(x)$ of $f_{0}$ in $\mathfrak{A}$. If $f_{0}$ has finite range $\left\{a_{1}, a_{2}, \cdots, a_{n}\right\}$, choose the action $x \rightarrow f_{0}(x)=\sum_{i=1}^{n} a_{i} e_{i}(x)$ where $e_{i}(x) e_{j}(x)=$ $\delta_{i j} e_{i}(x)\left(\delta_{i j}\right.$ the Kronecker delta). A standard Zorn's lemma argument shows that there exists a subalgebra $\mathscr{L}_{0}$ of $\mathscr{C}$ and a mapping $\varphi_{0}$ : $\mathscr{A}_{0} \times \mathfrak{A}_{1} \rightarrow \mathfrak{A}$ such that the pair $\left(\mathscr{C}_{0}, \varphi_{0}\right)$ is maximal in the set of all pairs $(\mathscr{L}, \psi)$ with the properties (1) $\mathscr{L}$ is a subalgebra of $\mathscr{C}$, $f_{0} \in \mathscr{L}$ and $\psi$ is a mapping from $\mathscr{C} \times \mathfrak{A}_{1}$ into $\mathfrak{A} ;(2) \psi\left(f_{0}, x\right)=f_{0}(x)$ for all $x \in \mathfrak{A}_{1}$; (3) for each $f \in \mathscr{L}$ the mapping $\psi(f, \cdot): \mathfrak{A}_{1} \rightarrow \mathfrak{U}$ is an action of $f$ in $\mathfrak{X}$; (4) for each $x \in \mathfrak{A}_{1}$ the mapping $\psi(\cdot, x): \mathscr{L} \rightarrow \mathfrak{U}$ is an algebra homomorphism on $\mathscr{L}$.

If $\mathfrak{U}$ is a commutative, semisimple Banach algebra, then the action $x \rightarrow f(x)$ of each $f \in \mathscr{M}$ is unique. In this case we define $\varphi: \mathscr{M} \times$ $\mathfrak{A}_{1} \rightarrow \mathfrak{A}$ by setting $\varphi(f, x)=f(x)$ for all $(f, x) \in \mathscr{M} \times \mathfrak{A}_{1}$.

In this way we see that if $f_{0}$ acts in $\mathfrak{A}$, then there is an algebra $\mathscr{M}_{0}$ of functions and a mapping $\varphi: \mathscr{M}_{0} \times \mathfrak{N}_{1} \rightarrow \mathfrak{A}$ such that $f_{0} \in \mathscr{M}_{0}$ and $\mathscr{C}_{0}$ and $\varphi$ satisfy conditions (2)-(4) above. Thus we can make a meaningful definition of an action of an algebra of functions in $\mathfrak{A}$.

DeFinition 3.12. An algebra $\mathscr{C}$ of functions defined on the unit disk $U$ acts in a Banach algebra $\mathfrak{A}$ if there exists a mapping $\varphi$ : $\mathscr{M} \times \mathfrak{N}_{1} \rightarrow \mathfrak{A}$ such that

(1) for each $f \in \mathscr{M}$, the mapping $\varphi(f, \cdot): \mathfrak{A}_{1} \rightarrow \mathfrak{A}$, is an action 
of $f$ in $\mathfrak{A}$;

(2) for each $x \in \mathfrak{A}_{1}$, the mapping $\varphi(\cdot, x): \mathscr{C} \rightarrow \mathfrak{A}$ is an algebra homomorphism on $\mathscr{M}$.

The mapping $\varphi$ is called an action of $\mathscr{M}$ in $\mathfrak{A}$.

EXAMPLE 3.13. Let $\mathfrak{A}$ be a Banach algebra with identity $e$ and $\mathscr{C}$ the algebra of functions analytic on the open disk $U$. The mapping $\varphi: \mathscr{C l} \times \mathfrak{A}_{1} \rightarrow \mathfrak{U}$ defined by setting

$$
\varphi(f, x)=\frac{1}{2 \pi i} \int_{|\zeta|=r} f(\zeta)(\zeta e-x)^{-1} d \zeta,
$$

where $(f, x) \in \mathscr{C} \times \mathfrak{A}_{1}$ and $1>r>\max \{|\lambda|: \lambda \in \sigma(x)\}$, is an action of $\mathscr{C}$ in $\mathfrak{A}$. If $\mathfrak{A}$ does not have an identity, let $\mathscr{L}_{0}$ be the algebra of analytic functions on $U$ which vanish at zero and restrict $\varphi$ to $\mathscr{C}_{0} \times$ $\mathfrak{A}_{1}$. This restriction is an action of $\mathscr{C}_{0}$ in $\mathfrak{X}$.

4. Preliminary properties for subalgebras of $C_{p}$. The concept of functions acting (or operating) in a Banach algebra was first presented for the group algebras of locally compact abelian groups. For these algebras a complete characterization was obtained of the functions which act in the algebra. The trace class of compact operators serves as a fairly manageable noncommutative analogue of these group algebras and hence a logical choice for a study of functions which act in noncommutative Banach algebras. The classes $C_{p}, 1 \leqq p \leqq \infty$, which have been studied in [1] and [8] are amenable because of the properties of compact operators (for example, a countable point spectrum with zero as the only limit point, finite index for every nonzero eigenvalue). If we restrict our attention further to subalgebras of $C_{p}$ which contain normal operators, then we can describe the elements in terms of their eigenvalues and orthogonal projections and we can obtain an explicit expression for the $C_{p}$-norm of each normal operator. With these properties available we are able to characterize those functions which act in certain classes of subalgebras of $C_{p}$. (In a later paper [2] we consider actions of functions in more general subalgebras of $C_{p}$.)

In this section we present notation, definitions and propositions which will be needed in the sequel.

If $T \in C_{\infty}$ is normal, then the index of $\lambda \in \sigma(T)$ is one, the spaces $\operatorname{ker}(\lambda I-T), \lambda \in \sigma(T)$, are pairwise orthogonal and for each $\lambda \in \sigma(T)$, $H$ is the orthogonal sum of the subspaces $\operatorname{ker}(\lambda I-T)$ and $(\lambda I-T) H$ [1, p. 563]. We call the orthogonal projection $E_{\lambda}(T)$ onto $\operatorname{ker}(\lambda I-T)$ the Riesz projection onto $\operatorname{ker}(\lambda I-T)$. For $\lambda \in \sigma(T), E_{\lambda}(T)$ is an element of the closed algebra generated in $C_{\infty}$ by $T[1$, p. 573]. If 
$\mathfrak{A}$ is a closed commutative subalgebra of $L(H)$ containing $T$, then $\left(E_{\lambda}(T)\right)^{\wedge}$ is the characteristic function of the set $\left\{h \in M_{\mathfrak{X}}: h(T)=\lambda\right\}$ $(\S 2)$. It is an easily checked consequence of the spectral theorem for normal operators (see p. 897-899 of [1]) that if $T \in C_{\infty}$ is normal, then $T=\sum_{\sigma(T)} \lambda E_{\lambda}(T)$, with convergence in the operator norm.

Suppose $T \in C_{\infty}$ and $\mu_{1}(T), \mu_{2}(T), \cdots$ are the distinct nonzero eigenvalues of the self-adjoint operator $\sqrt{T^{*} T}$. Let $k_{n}$ be the dimension of $\operatorname{ker}\left(\mu_{n}(T) I-\sqrt{T^{*} T}\right)$. For each $p \in[1, \infty)$ the set $C_{p}=\{T \in$ $\left.C_{\infty}: \sum_{n=1}^{\infty}\left(\mu_{n}(T)\right)^{p} k_{n}<\infty\right\}$ is a $*$-subalgebra of $C_{\infty}$ which contains the algebra $C_{0}$ of operators of finite rank. Setting

$$
|T|_{p}=\left[\sum_{n=1}^{\infty}\left(\mu_{n}(T)\right)^{p} k_{n}\right]^{1 / p}
$$

for each $T \in C_{p}, 1 \leqq p<\infty$, and $|T|_{\infty}=\|T\|$ for $T \in C_{\infty}$ defines a norm on $C_{p}, 1 \leqq p<\infty$, which has the following properties:

(1) $|T|_{p}=\left|T^{*}\right|_{p}=\left|\sqrt{T^{*} T}\right|_{p}$ for $1 \leqq p \leqq \infty$;

(2) $|T S|_{p} \leqq\|S\||T|_{p}$ and $|S T|_{p} \leqq\|S\||T|_{p}$ for all $S \in L(H), T \in C_{p}$;

(3) $|T|_{q} \leqq|T|_{p}$ for all $T \in C_{p}, 1 \leqq p \leqq q \leqq \infty$.

The algebra $C_{p}$ is complete with respect to the norm $|\cdot|_{p}[1$, p. 1088 ff: 8 ].

If $T \in C_{\infty}$ is normal, then $\sqrt{T^{*} T}=\sum_{\sigma(T)}|\lambda| E_{\lambda}(T)$ and the dimension of $\operatorname{ker}\left(\mu_{j} I-\sqrt{T^{*} T}\right)$ is the sum $\sum\left\{\operatorname{dim} E_{\lambda}(T) H: \lambda=\mu_{j}\right\}$. Thus a normal, compact operator $T$ is in $C_{p}, 1 \leqq p<\infty$, if, and only if, $\sum_{\sigma(T)}|\lambda|^{p}\left(\operatorname{dim} E_{\lambda}(T) H\right)<\infty$, in which case

$$
|T|_{p}=\left[\sum_{\sigma(T)}|\lambda|^{p} \operatorname{dim} E_{\lambda}(T) H\right]^{1 / p} .
$$

We denote by $\mathfrak{U}_{p}(T)$ the closed subalgebra of $C_{p}$ generated by $T \in C_{p}$.

The spectrum of $T$ in $C_{p}$ is again the set $\sigma(T)$ [1, p. 1014]. If $\mathfrak{A}$ is a closed subalgebra of $C_{p}, 1 \leqq p \leqq \infty$, then $\sigma_{\mathfrak{A}}(T)=\sigma(T)$ because the spectrum of $T$ does not separate the plane [11, p. 34]. For every operator $T \in C_{p}$ we identify $\sigma_{0}(T)$ and the maximal ideal space of $\mathfrak{U}_{p}(T)$.

Lemma 4.1. Let $T \in C_{p}$ be normal with $\sigma_{0}(T)=\left\{\lambda_{1}, \lambda_{2}, \cdots\right\}, E_{j}$ the Riesz projection onto $\operatorname{ker}\left(\lambda_{j} I-T\right)$ and $k_{j}=\operatorname{dim} E_{j} H$. Then $S \in \mathfrak{A}_{p}(T)$ if, and only if, $S=\sum_{j=1}^{\infty} \hat{S}\left(\lambda_{j}\right) E_{j}$ and $\sum_{j=1}^{\infty}\left|\widehat{S}\left(\lambda_{j}\right)\right|^{p} k_{j}<\infty$ if $1 \leqq p<\infty$ or $\lim _{j \rightarrow \infty} \widehat{S}\left(\lambda_{j}\right)=0$ if $p=\infty$.

Proof. Suppose $S=\sum_{j=1}^{\infty} \mu_{j} E_{j}$ with $\sum_{j=1}^{\infty}\left|\mu_{j}\right|^{p} k_{j}<\infty$ if $1 \leqq p<$ $\infty$ or $\lim _{j \rightarrow \infty} \mu_{j}=0$ if $p=\infty$. Then $\lim _{n \rightarrow \infty}\left|S-\sum_{j=1}^{n} \mu_{j} E_{j}\right|_{p}=0$ so that $S$ is the limit of the sequence $\left\langle\sum_{j=1}^{n} \mu_{j} E_{j}\right\rangle \subset \mathfrak{A}_{p}(T)$. Thus $S \in \mathfrak{A}_{p}(T)$ with $\hat{S}\left(\lambda_{j}\right)=\mu_{j}$. Conversely, if $S \in \mathfrak{A}_{p}(T)$, then $S \in \mathfrak{A}_{\infty}(T)$ so that 
$S=\sum_{j=1}^{\infty} \hat{S}\left(\lambda_{j}\right) E_{j}$ as a consequence of the spectral theorem for normal operators [1, pp. 897-899]. The sum or limit condition follows from the fact that $S \in \mathfrak{A}_{p}(T)$.

Our second lemma allows us to construct normal operators in $C_{p}$ with given multiplicities for their eigenvalues.

LEMMA 4.2. Suppose $\left\langle k_{n}\right\rangle$ is a sequence of positive integers, $p \in$ $[1, \infty), H$ an infinite-dimensional Hilbert space and $\left\langle\lambda_{n}\right\rangle$ a sequence of nonzero complex numbers such that $\sum_{n=1}^{\infty}\left|\lambda_{n}\right|^{p} k_{n}<\infty$ if $1 \leqq p<\infty$ or $\lim _{n \rightarrow \infty} \lambda_{n}=0$ if $p=\infty$. Then there exists a normal operator $T \in C_{p}$ such that $\sigma_{0}(T)=\left\{\lambda_{n}: n=1,2, \cdots\right\}$ and $\operatorname{dim} \operatorname{ker}(\lambda I-T)=\sum\left\{k_{j}: \lambda_{j}=\right.$ $\lambda\}$ for each $\lambda \in \sigma_{0}(T)$. If $\left\langle P_{n}\right\rangle$ is a sequence of pairwise orthogonal self-adjoint projections and $\operatorname{dim} P_{n} H=k_{n}$, then the operator $T=$ $\sum_{n=1}^{\infty} \lambda_{n} P_{n}$ has the desired properties. For each sequence $\left\langle k_{n}\right\rangle$ of nonzero integers there exists a normal operator $T=\sum_{n=1}^{\infty} \lambda_{n} P_{n} \in C_{p}(1 \leqq$ $p \leqq \infty)$ such that $\left\langle P_{n}\right\rangle$ is a family of pairwise orthogonal self-adjoint projections, $\left|\lambda_{n}\right|>\left|\lambda_{n+1}\right|>0$ and $\operatorname{dim} P_{n} H=K_{n}=\operatorname{dim} \operatorname{ker}\left(\lambda_{n} I-T\right)$ $(n=1,2, \cdots)$.

Since the proof of the lemma is straightforward, it is left to the reader.

If $\mathfrak{A}$ is a closed subalgebra of $C_{p}$ and $T \in \mathfrak{A}$ is normal, then $T^{*} \in \mathfrak{A}$ (Lemma 4.1) and whenever $f(T)$ is defined, $f(T)$ is normal. The following proposition establishes a useful expression for $f(T)$ in terms of the Riesz projections $E_{\lambda}(T)$.

Proposition 4.3. Let $\mathfrak{X}$ be a closed subalgebra of $C_{p}$, T a normal operator in $\mathfrak{X}$, and $f$ a function which defines an action $S \rightarrow f(S)$ in भi. If $S \in \mathfrak{U}_{p}(T) \cap \mathfrak{N}_{1}$, then $f(S)=\sum_{n=1}^{\infty} f\left(\widehat{S}\left(\lambda_{n}\right)\right) E_{\lambda_{n}}(T)$ and $|f(S)|_{p}^{p}=$ $\sum_{n=1}^{\infty} \mid f\left(\left.\hat{S}\left(\lambda_{n}\right)\right|^{p} \operatorname{dim} E_{\lambda_{n}}(T) H\right.$.

Proof. Suppose $S \in \mathfrak{A}_{1} \cap \mathfrak{A}_{p}(T), \sigma_{0}(T)=\left\{\lambda_{1}, \lambda_{2}, \cdots\right\}$ and $E_{n}$ is the Riesz projection onto ker $\left(\lambda_{n} I-T\right)$. Since $S$ is normal, $f(S)$ is normal (Corollary 3.7.1) with

$$
f(S)=\sum\left\{\mu E_{\mu}(f(S)): \mu \in \sigma_{0}(f(S))\right\}
$$

and $\sigma(f(S))=\left\{f\left(\hat{S}\left(\lambda_{n}\right)\right): n=1,2, \cdots\right\} \cup\{0\}$ (Proposition 3.5 and Lemma 4.1). Therefore $f(S)^{*} \in \mathfrak{A}$ and there exists a maximal commutative. *-subalgebra $\mathscr{C}$ of $\mathfrak{A}$ such that $S \in \mathscr{C}$ and hence $f(S) \in \mathscr{C}$ (Definition 3.1). The projections $E_{\mu}(f(S)), \mu \in \sigma_{0}(f(S))$, and $E_{n}, n=1,2, \cdots$, are in $\mathscr{C}$. Moreover, $\left[E_{\mu}(f(S))\right]^{\wedge}$ is the characteristic function of the set 
$\left\{h \in M_{\mathscr{\&}}: h(f(S))=\mu\right\} \quad(\mu \neq 0)$ but the function $\sum\left\{E_{n}^{\wedge}: f\left(\widehat{S}\left(\lambda_{n}\right)\right)=\mu\right\}$ also is the characteristic function of this set. Since $\mathscr{C}$ is semisimple [10, p. 309], we have $E_{\mu}(f(S))=\sum\left\{E_{n}: f\left(\hat{S}\left(\lambda_{n}\right)\right)=\mu\right\}$ for each $\mu \epsilon$ $\sigma_{0}(f(S))$. On rearranging terms in (4.2) we obtain $f(S)=\sum_{n=1}^{\infty} f\left(\hat{S}\left(\lambda_{n}\right)\right) E_{n}$. The equation for the $C_{p}$-norm of $f(S)$ follows from this equation and Lemma 4.1.

5. Functions with actions in subalgebras of $C_{p}$. Suppose $\mathfrak{Y}$ is a subalgebra of $C_{p}$ which contains only normal operators of finite rank. Let $f$ be any function defined on $U$ with $f(0)=0$ if $\operatorname{dim} H=\infty$. For $T \in \mathfrak{A}_{1}$ set $f(T)=\sum\left\{f(\lambda) E_{\lambda}(T): \lambda \in \sigma_{0}(T)\right\}$. Then the mapping $T \rightarrow f(T)$, $T \in \mathfrak{N}_{1}$, is an action of $f$ in $\mathfrak{U}$. Thus we see that if $\mathfrak{X}$ contains only normal operators of finite rank there are no restrictions on functions which can act in $\mathfrak{U}$ except the condition that $f(0)=0$ if $\operatorname{dim} H=\infty$ which is necessary if $\mathfrak{U}$ contains no nonzero central idempotents.

The case when $\mathfrak{U}$ contains a normal operator of infinite rank is of more interest. Our first proposition shows that not all functions can act in a subalgebra of $C_{p}$ which contains a normal operator of infinite rank.

Proposition 5.1. If $f$ acts in a closed subalgebra il of $C_{p}, 1 \leqq$ $p \leqq \infty$, and if $\mathfrak{X}$ contains a normal operator of infinite rank, then $f$ is continuous at zero and $f(0)=0$.

Proof. Let $T \in \mathfrak{X}$ be a normal operator such that $\sigma_{0}(T)=\left\{\lambda_{1}\right.$, $\left.\lambda_{2}, \cdots\right\}$ is infinite. Let $E_{n}$ denote the Riesz projection onto $\operatorname{ker}\left(\lambda_{n} I-T\right)$.

Suppose $\lim _{z \rightarrow 0} f(z) \neq 0$. Then there exists $\varepsilon>0$ and a sequence $\left\langle z_{n}\right\rangle$ such that $\left|z_{n}\right|<\left(n^{2} k_{n}\right)^{-1 / p}\left(k_{n}=\operatorname{dim} E_{n} H\right)$ if $1 \leqq p<\infty$ or $\left|z_{n}\right|<$ $1 / n$ if $p=\infty$ and $\left|f\left(z_{n}\right)\right| \geqq \varepsilon$ for $n=1,2, \cdots$. The sum $\sum_{n=1}^{\infty} z_{n} E_{n}$ defines an element $S$ of $\mathfrak{I}_{p}(T)$ with $\sigma_{0}(S)=\left\{z_{1}, z_{2}, \cdots\right\}$ (Lemma 4.1 and Lemma 4.2). Thus $f(S)$ is defined and $f(S)=\sum_{n=1}^{\infty} f\left(z_{n}\right) E_{n}$ (Proposition 4.3). But for any integer $N>0$ we have $|f(S)|_{p}^{p}=\sum_{n=1}^{\infty}\left|f\left(z_{n}\right)\right|^{p} k_{n} \geqq$ $\varepsilon^{p} N$ if $1 \leqq p<\infty$ and $|f(S)|_{\infty} \geqq\left|f\left(z_{n}\right)\right| \geqq \varepsilon(n=1,2, \cdots)$ if $p=\infty$. Since $f(S) \in \mathfrak{N}_{p}(T)$ we arrive at a contradiction (Lemma 4.1). Thus $\lim _{z \rightarrow 0} f(z)=0$.

Choose $T \in \mathfrak{X}_{1}$ such that $T$ is normal and of infinite rank. Then $f(T) \in \mathfrak{V}_{p}(T)$ (Proposition 4.3). Hence $f(T)$ is continuous at zero with $(f(T))^{\wedge}(0)=0$. But if $f(0) \neq 0$, we would have a contradiction.

Only for a restricted class of subalgebras of $C_{\infty}$ can we obtain a. converse to Proposition 5.1.

THEOREM 5.2. Let 2 be a closed subalgebra of $C_{\infty}$ such that the elements of $\mathfrak{X}$ are normal operators. Suppose $\mathfrak{A}$ contains an operator 
of infinite rank. Then $f$ acts in 2 if, and only if, $f$ is continuous at zero and $f(0)=0$.

Proof. We have already seen that if $f$ acts in $\mathfrak{A}$, then $f(0)=0$ and $f$ is continuous at zero (Proposition 5.1). If $f$ is continuous at zero with $f(0)=0$, then for each $T \in \mathfrak{P}_{1}, f \circ T$ is in $C_{0}(\sigma(T)$ ), an algebra which is isomorphic to $\mathfrak{V}_{\infty}(T)$ under the Gelfand mapping. Hence there exists $f(T) \in \mathfrak{N}_{\infty}(T)$ such that $(f(T))^{\wedge}=f \circ \hat{T}$ on $\sigma_{0}(T)$ and, in fact, $f(T)=\sum\left\{f(\lambda) E_{\lambda}(T): \lambda \in \sigma_{0}(T)\right\}$. It is easily checked that the mapping $T \rightarrow f(T), T \in \mathfrak{A}_{1}$, defined in this way is an action of $f$ in $\mathfrak{A}$.

The preceding theorem completely characterizes those functions which act in a closed subalgebra of $C_{\infty}$ which contains only normal operators if one of those operators has infinite rank. The corresponding problem for $C_{p}, 1 \leqq p<\infty$, is not as simple as we shall see. We can, however, show the existence of actions for a much larger class of functions than those analytic on the unit disk.

A function $f$ is said to be Hölder-continuous at $z_{0}$ if there exist positive constants $B$ and $\delta$ (both depending on $z_{0}$ ) such that $\mid f(z)-$ $f\left(z_{0}\right)|\leqq B| z-z_{0} \mid$ whenever $z \in U$ and $\left|z-z_{0}\right|<\delta$. For the algebra of functions which are Hölder-continuous at zero we have the following proposition.

Proposition 5.3. Let $\mathfrak{i}$ be a closed subalgebra of $C_{p}(1 \leqq p<\infty)$ which contains only normal operators. If $f$ is defined on the unit disk and Hölder-continuous at zero with $f(0)=0$, then $f$ acts in $\mathfrak{Y}$.

Proof. If $f$ is Hölder-continuous at zero with $f(0)=0$, then there exist constants $B>0, \delta>0$ such that $|f(z)| \leqq B|z|$ whenever $|z| \leqq \delta$. Suppose $T \in \mathfrak{V}_{1}$ with $\sigma_{0}(T)=\left\{\lambda_{1}, \lambda_{2}, \cdots\right\}, E_{n}=E_{\lambda_{n}}(T)$ and $k_{n}=\operatorname{dim} E_{n} H$. Since

$$
\sum_{n=1}^{\infty}\left|f\left(\lambda_{n}\right)\right|^{p} k_{n} \leqq \sum_{\left|\lambda_{n}\right| \geqq \delta}\left|f\left(\lambda_{n}\right)\right|^{p} k_{n}+B^{p} \sum_{n=1}^{\infty}\left|\lambda_{n}\right|^{p} k_{n}<\infty,
$$

the operator $f(T)=\sum_{n=1}^{\infty} f\left(\lambda_{n}\right) E_{n}$ is in $\mathfrak{N}_{p}(T)$ (Lemma 4.1). It is easily checked that the mapping that takes $T=\sum_{n=1}^{\infty} \lambda_{n} E_{n}$ into $f(T)=$ $\sum_{n=1}^{\infty} f\left(\lambda_{n}\right) E_{n}\left(T \in \mathfrak{U}_{1}\right)$ is an action of $f$ in $\mathfrak{Q}$.

Our main result in characterizing those functions acting in closed subalgebras of $C_{p}$ which contain only normal operators in a sense completes Proposition 5.3 and we get that the functions which operate in a restricted class of subalgebras of $C_{p}$ are exactly those which vanish at zero and are Hölder-continuous at zero.

TheOREM 5.4. Let $\mathfrak{A}$ be a closed subalgebra of $C_{p}(1 \leqq p<\infty)$ 
such that the elements of $\mathfrak{X}$ are all normal. Suppose there exists $T \in \mathfrak{A}$ with $\sigma_{0}(T)=\left\{\lambda_{1}, \lambda_{2}, \cdots\right\}$ and constants $K_{1}, K_{2}>1$ and $r_{1}, r_{2}>0$ such that

$$
K_{1}^{r_{1}(n+1)}>K_{2}^{r_{2} n}
$$

and

$$
K_{1^{r_{1} n}}^{r} \leqq \operatorname{dim} \operatorname{ker}\left(\lambda_{n} I-T\right) \leqq K_{2}^{r_{2}(n+1)}
$$

for $n=1,2, \cdots$. Then a necessary and sufficient condition for the existence of an action of a function $f$ in $\mathfrak{A}$ is that $f$ be Höldercontinuous at zero with $f(0)=0$.

Proof. The sufficiency of Hölder-continuity at zero with $f(0)=0$ is proved in Proposition 5.3. We prove the necessity of this condition.

Suppose $T \in \mathfrak{X}$ satisfies the hypothesis of the theorem and $E_{n}=$ $E_{\lambda_{n}}(T)$. Since $T$ is a normal operator of infinite rank, $f(0)=0$ (Proposition 5.1).

If $f$ is not Hölder-continuous and $f$ acts in $\mathfrak{A}$, then there exists a sequence $\left\langle z_{n}\right\rangle \subset U$ such that

$$
\begin{gathered}
\left|z_{1}\right|<K_{2}^{-r_{2} / p} \text { and }\left|z_{n}\right|<\left|z_{n-1}\right|\left(\frac{n-1}{n}\right)^{(p+1) / p} K_{2}^{-r_{2} / p}(n \geqq 2) \\
\left|f\left(z_{n}\right)\right|>n\left|z_{n}\right| \text { for } n=1,2, \cdots
\end{gathered}
$$

Define $m_{n}$ to be the largest integer not greater than

$$
\frac{-1}{r_{2}} \log _{K_{2}}\left(\left|z_{n}\right|^{p} n^{p+1}\right) \text {. }
$$

As a consequence of (5.5) $m_{n}$ is a strictly increasing sequence of positive integers. Set $R=\sum_{n=1}^{\infty} z_{n} E_{m_{n}}$. As a result of inequality (5.4) with $m_{n}$ replacing $n, R \in \mathfrak{N}$ (Lemma 4.2). Thus $f(R)$ is in $\mathfrak{A}$ and $f(R)=\sum_{n=1}^{\infty} f\left(z_{n}\right) E_{m_{n}}$ (Proposition 4.3). But from condition (5.3) we obtain $r_{1} / r_{2} \geqq \log _{K_{1}} K_{2}$ and hence

$$
\begin{aligned}
|f(R)|_{p}^{p} & >\sum_{n=1}^{\infty}\left(n\left|z_{n}\right|\right)^{p} K_{1}^{r_{1} m_{n}} \\
& \geqq K_{1}^{-r_{1}} \sum_{n=1}^{\infty}\left(n\left|z_{n}\right|\right)^{p} K_{1}^{\left(-r_{1} \mid r_{2}\right) \log _{3} K_{2}\left(\left|z_{n}\right| p_{n} p+1\right)} \\
& \geqq K_{1}^{-r_{1}} \sum_{n=1}^{\infty}\left(n\left|z_{n}\right|\right)^{p} K_{1}^{\left(-\log _{K_{1}} K_{2}\right) \log _{K_{2}}\left(\left|z_{n}\right| p_{n} p+1\right)} \\
& =K_{1}^{-r_{1}} \sum_{n=1}^{\infty} \frac{1}{n}
\end{aligned}
$$


which is an obvious contradiction. Therefore, $f$ must be Höldercontinuous at zero.

It is clear that the hypothesis of Theorem 5.4 is satisfied if $\mathfrak{A}$ contains an operator $T$ with $\sigma_{0}(T)=\left\{\lambda_{1}, \lambda_{2}, \cdots\right\}$ such that $\operatorname{dim} \operatorname{ker}\left(\lambda_{n} I-\right.$ $T)=K^{r n}$ for some integers $K \geqq 2$ and $r \geqq 1$. For the case of an operator with bounded multiplicities we have the following corollary.

COROLLARY 5.4.1. Let $\mathfrak{A}$ be a closed subalgebra of $C_{p}(1 \leqq p<\infty)$ which contains only normal operators. Suppose $T \in \mathfrak{A}$ and that the sequence $\left\{\operatorname{dim} \operatorname{ker}(\lambda I-T): \lambda \in \sigma_{0}(T)\right\}$ is uniformly bounded. Then a function $f$ acts in $\mathfrak{A}$ if, and only if, $f$ is Hölder-continuous at zero with $f(0)=0$.

Proof. Choose $T \in \mathfrak{A}$ such that $\sigma_{0}(T)=\left\{\lambda_{1}, \lambda_{2}, \cdots\right\}$ and $\left\langle\operatorname{dim} \operatorname{ker}\left(\lambda_{n} I-\right.\right.$ $T)\rangle$ is a bounded sequence. Set $k_{n}=\operatorname{dim} \operatorname{ker}\left(\lambda_{n} I-T\right)$ and choose $K \geqq 2$ such that $K \geqq k_{n}$ for $n=1,2, \cdots$. Then we can choose by induction two sequences $\left\langle r_{n}\right\rangle$ and $\left\langle m_{n}\right\rangle$ of integers such that $m_{n}=$ $\sum\left\{k_{j}: r_{n-1}<j \leqq r_{n}\right\}$ and $K^{n+1}-K<m_{n} \leqq K^{n+1}$. Set $P_{n}=\sum\left\{E_{j}\right.$ : $\left.r_{n-1}<j \leqq r_{n}\right\}$, where $E_{n}=E_{\lambda_{n}}(T)$. Then there exists $R \in \mathfrak{A}$ such that $R=\sum_{n=1}^{\infty} z_{n} P_{n}, \sigma_{0}(R)=\left\{z_{1}, z_{2}, \cdots\right\}$ and $\operatorname{dim} \operatorname{ker}\left(z_{n} I-R\right)=m_{n}$ (Lemma 4.2). This operator $R$ satisfies the hypotheses of Theorem 5.4.

In studying the conditions of Theorem 5.4 one might ask whether the bounds of (5.4) are as strong as possible. The following examples show that the upper bound cannot be altered much before a larger class of functions can act in $\mathfrak{A}$ nor can the lower bound be omitted.

The hypothesis of Theorem 5.4 is satisfied by all subalgebras of $C_{p}$ which contain normal operators, one of which has multiplicities $k_{n}=2^{n^{2}}$ for $n=1,2, \cdots, N$ and $k_{n}=2^{r n}$ for some fixed $r>1$ and all $n \leqq N$. The theorem, however, is no longer true if $\mathfrak{A}$ is the algebra generated by a normal operator $T \in C_{p}$ with $\sigma_{0}(T)=\left\{\lambda_{1}, \lambda_{2}, \cdots\right\}$ and $\operatorname{dim} \operatorname{ker}\left(\lambda_{n} I-T\right)=2^{n^{2}}$ for $n=1,2, \cdots$.

To see this let $f$ be the function defined on the unit disk by setting $f(z)=0$ for $|z| \neq 2^{-n^{2} / p}$ and $f(z)=g(n)|z|$ for $|z|=2^{-n^{2} / p}$, where $\langle g(n)\rangle$ is an unbounded sequence of positive numbers and $\lim _{n \rightarrow \infty} g(n) 2^{-n^{2} / p}=0$. Then $f$ is continuous at zero but not Hölder-continuous at zero.

If the sequence $\left\langle g(n)^{p 2^{-2 n}}\right\rangle$ is summable then $f$ acts in $\mathfrak{X}$. To prove this we need only consider operators $S \in \mathfrak{A}$ such that $S=\sum_{n=1}^{\infty} z_{n} E_{m_{n}}$, where $E_{n}$ is the Riesz projection onto $\operatorname{ker}\left(\lambda_{n} I-T\right),\left|z_{n}\right|=2^{-r_{n}^{2} / p}$ for some positive integers $r_{n}$, and $m_{1}<m_{2}<\cdots$. Since $S \in \mathfrak{A}$, there exists $N>0$ such that $r_{n}-m_{n} \geqq 1$ for $n \geqq N$ (Lemma 4.1) and we can assume without loss of generality that this inequality is true for all 
n. But then we have

$$
\sum_{n=1}^{\infty}\left|f\left(z_{n}\right)\right|^{2^{m^{m_{n}^{2}}}} \leqq 2 \sum_{n=1}^{\infty}(g(n))^{p^{2^{-2 n}}}<\infty .
$$

On the other hand, if the sequence $\left\langle g(n)^{p^{2-2 n}}\right\rangle$ is not summable, then $f$ does not act in $\mathfrak{A}$. To see this let $z_{n}=2^{-(n+1)^{2} / p}$ and $S=$ $\sum_{n=1}^{\infty} z_{n} E_{n}$. Then $S$ is in $\mathfrak{A}$ (Lemma 4.1) but

$$
\sum_{n=1}^{\infty}\left|f\left(z_{n}\right)\right|^{2^{2^{n^{2}}}}=\sum_{n=1}^{\infty}(g(n))^{p^{2^{-(n+1)^{2}}}} 2^{n^{2}}=\frac{1}{2} \sum_{n=1}^{\infty} 2^{-2 n}(g(n))^{p}=\infty
$$

so that $f(S)$ cannot be in $C_{p}$ (if it were, then $f(S)$ would be the operator $\sum_{n=1}^{\infty} f\left(z_{n}\right) E_{n}$ as a consequence of Proposition 4.3).

Now suppose we delete the lower bound condition of Theorem 5.4. In this case we construct an algebra and a function $f$ such that the multiplicities of elements of the algebra are bounded above by $2^{n}$ and $f$ acts in the algebra and is not Hölder-continuous.

Denote by $[n]$ the integer in the interval $(\sqrt{n}-1, \sqrt{n}]$. Let $T$ be a normal operator in $C_{p}(1 \leqq p<\infty)$ such that $\sigma_{0}(T)=\left\{\lambda_{1}, \lambda_{2}, \cdots\right\}$, and $k_{n}=\operatorname{dim} \operatorname{ker}\left(\lambda_{n} I-T\right)=[n]^{2}$ (Lemma 4.2 ensures that $T$ exists). For each integer $n$ we have $k_{n} \leqq 2^{n}$ but $k_{n^{2}-1}=2^{(n-1)^{2}}<2^{n^{2-1}}$ for $n=2,3, \cdots$.

Let $\mathfrak{A}$ be the algebra $\mathfrak{U}_{p}(T)$. Denote by $E_{n}$ the Riesz projection onto $\operatorname{ker}\left(\lambda_{n} I-T\right)$. Define $f(z)$ to be 0 for $|z| \neq 2^{-n^{2} / p}$ and $\left(n 2^{-n^{2}}\right)^{1 / p}$ for $|z|=2^{-n^{2} / p}$. Obviously, $f$ is continuous, but not Hölder-continuous, at zero.

As before we need consider only operators $S \in \mathfrak{A}$ such that $S=$ $\sum_{n=1}^{\infty} z_{n} E_{m_{n}}$ with $\left|z_{n}\right|=2^{-r_{n}^{2} / p}$. If $S \in \mathfrak{R}_{1}$, we must have $r_{n} \geqq\left[m_{n}\right]+1$ for all large $n$ and we can assume the inequality is true for all $n>1$. Since the function $g(t)=t 2^{-t^{2}}$ is decreasing for $t \geqq 0$ and there are $2 n+1$ integers in the sequence $\left\langle k_{j}\right\rangle$ equal to $2^{n^{2}}$, we have

$$
\begin{aligned}
\sum_{n=1}^{\infty}\left|f\left(z_{n}\right)\right|^{p} k_{m_{n}} & \leqq \sum_{n=1}^{\infty}\left(\left[m_{n}\right]+1\right) 2^{-\left(\left[m_{n}\right]+1\right)^{2}} 2^{\left[m_{n}\right]^{2}} \\
& \leqq \sum_{q=1}^{\infty} 2^{-2 q}(2 q+1)(q+1)<\infty .
\end{aligned}
$$

In view of Lemma 4.1 the operator $f(S)=\sum_{n=1}^{\infty} f\left(z_{n}\right) E_{m_{n}}$ is in $\mathfrak{X}$.

We have determined the algebra of functions which acts in certain classes of subalgebras of $C_{p}(1 \leqq p \leqq \infty)$. The problem of characterizing those functions which act in other classes, however, is still open. For example, which functions act in the algebras $\mathfrak{U}_{p}(T)$ if $T$ is a normal operator with multiplicities $k^{n^{2}}$ for some $k \geqq 2$ ?

We wish to thank Professors J. Gil de Lamadrid and C. A. McCarthy 
for their suggestions and encouragement while this research was being carried out.

\section{BIBLIOGRAPHY}

1. N. Dunford and J. T. Schwartz, Linear operators, Parts I and II, Interscience Publishers, Inc., New York, 1958, 1966.

2. F. R. Gantmacher, The theory of matrices (tr. by K. A. Hirsch), Chelsea Publishing Co., New York, 1959.

3. F. F. Gulick, Derivations and the action of functions, (to appear in Pacific J. Math.)

4. H. Helson, J. P. Kahane, Y. Katznelson, and W. Rudin, The functions which operate on Fourier transforms, Acta Math. 102 (1959), 135-137.

5. Y. Katznelson, Sur le calcul symbolique dans quelques algebres de Banach, Ann. Sci. Ecole Norm. Sup. 76 (1959), 83-124.

6. P. Levy, Sur les convergence absolue des series de Fourier, Compositio Math. 1 (1934), 1-14.

7. L. H. Loomis, An introduction to abstract harmonic analysis, Van Nostrand, Princeton, 1953.

8. C. A. McCarthy, $c_{p}$, Israel J. Math. 5 (1967), 249-271.

9. E. A. Michael, Locally multiplicatively-convex topological algebras, Mem. Amer. Math. Soc. 11 (1952).

10. N. Naimark, Normed rings, P. Noordhoff, N. V. Groningen, 1964.

11. C. E. Rickart, General theory of Banach algebras, Van Nostrand, Princeton, 1960.

12. Riesz and B. Sz-Nagy, Functional analysis, F. Ungar Publishing Co., New York, 1956.

13. W. Rudin, Fouries analysis on groups, Interscience Publishers, New York, 1962.

14. N. Wiener, Tauberian theorems, Ann. of Math. 33 (1932), 1-100.

Received February 20, 1969, and in revised form February 11, 1970. This paper is drawn from the author's doctoral thesis written under the supervision of Professor J. Gil de Lamadrid (University of Minnesota, 1968). This work was supported in part by the National Science Foundation.

UNIVERSITY OF MARYLAND AND

The Catholic University of America 



\section{PACIFIC JOURNAL OF MATHEMATICS}

\section{EDITORS}

H. SAMELSON

Stanford University

Stanford, California 94305

\section{Richard Pierce}

University of Washington

Seattle, Washington 98105
J. DugundJI

Department of Mathematics

University of Southern California

Los Angeles, California 90007

RICHARD ARENS

University of California

Los Angeles, California 90024

\section{ASSOCIATE EDITORS}

\section{E. F. BeCKenBACH}

B. H. NeUmanN
K. YosHida

\section{SUPPORTING INSTITUTIONS}

\author{
UNIVERSITY OF BRITISH COLUMBIA \\ CALIFORNIA INSTITUTE OF TECHNOLOGY \\ UNIVERSITY OF CALIFORNIA \\ MONTANA STATE UNIVERSITY \\ UNIVERSITY OF NEVADA \\ NEW MEXICO STATE UNIVERSITY \\ OREGON STATE UNIVERSITY \\ UNIVERSITY OF OREGON \\ OSAKA UNIVERSITY \\ UNIVERSITY OF SOUTHERN CALIFORNIA
}

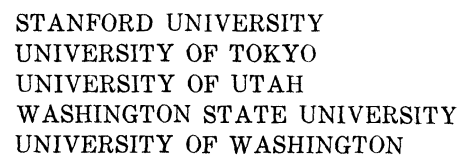

STANFORD UNIVERSITY UNIVERSITY OF TOKYO

UNIVERSITY OF UTAH

WASHINGTON STATE UNIVERSITY

UNIVERSITY OF WASHINGTON

The Supporting Institutions listed above contribute to the cost of publication of this Journal, but they are not owners or publishers and have no responsibility for its content or policies.

Mathematical papers intended for publication in the Pacific Journal of Mathematics should be in typed form or offset-reproduced, (not dittoed), double spaced with large margins. Underline Greek letters in red, German in green, and script in blue. The first paragraph or two must be capable of being used separately as a synopsis of the entire paper. The editorial "we" must not be used in the synopsis, and items of the bibliography should not be cited there unless absolutely necessary, in which case they must be identified by author and Journal, rather than by item number. Manuscripts, in duplicate if possible, may be sent to any one of the four editors. Please classify according to the scheme of Math. Rev. Index to Vol. 39. All other communications to the editors should be addressed to the managing editor, Richard Arens, University of California, Los Angeles, California, 90024.

50 reprints are provided free for each article; additional copies may be obtained at cost in multiples of 50 .

The Pacific Journal of Mathematics is published monthly. Effective with Volume 16 the price per volume (3 numbers) is $\$ 8.00$; single issues, $\$ 3.00$. Special price for current issues to individual faculty members of supporting institutions and to individual members of the American Mathematical Society: $\$ 4.00$ per volume; single issues $\$ 1.50$. Back numbers are available.

Subscriptions, orders for back numbers, and changes of address should be sent to Pacific Journal of Mathematics, 103 Highland Boulevard, Berkeley, California, 94708.

PUBLISHED BY PACIFIC JOURNAL OF MATHEMATICS, A NON-PROFIT CORPORATION

Printed at Kokusai Bunken Insatsusha (International Academic Printing Co., Ltd.), 7-17, Fujimi 2-chome, Chiyoda-ku, Tokyo, Japan. 


\section{Pacific Journal of Mathematics}

\section{Vol. 34, No. 3 \\ July, 1970}

Richard Hindman Bouldin, The peturbation of the singular spectrum

Hugh D. Brunk and Søren Glud Johansen, A generalized Radon-Nikodym derivative .

Henry Werner Davis, F. J. Murray and J. K. Weber, Families of $L_{p}$-spaces

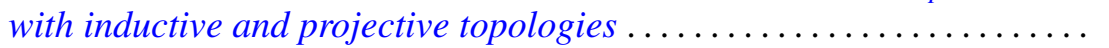

Esmond Ernest Devun, Special semigroups on the two-cell .

Murray Eisenberg and James Howard Hedlund, Expansive automorphisms

of Banach spaces ......................................

Frances F. Gulick, Actions of functions in Banach algebras.

Douglas Harris, Regular-closed spaces and proximities.

Norman Lloyd Johnson, Derivable semi-translation planes . .

Donald E. Knuth, Permutations, matrices, and generalized Young

tableaux..........................................

Herbert Frederick Kreimer, Jr., On the Galois theory of separable

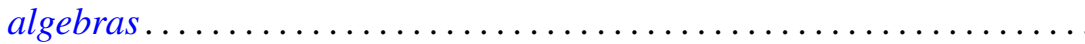

You-Feng Lin and David Alon Rose, Ascoli's theorem for spaces of

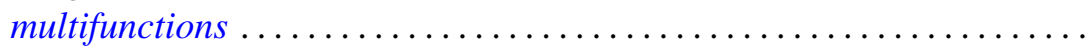

David London, Rearrangement inequalities involving convex functions . . . .

Louis Pigno, A multiplier theorem.

749

Helga Schirmer, Coincidences and fixed points of multifunctions into trees.

755

Richard A. Scoville, Some measure algebras on the integers .

Ralph Edwin Showalter, Local regularity of solutions of Sobolev-Galpern

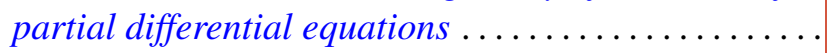

Allan John Sieradski, Twisted self-homotopy equivalences

John H. Smith, On S-units almost generated by S-units of subfields ...

803

Masamichi Takesaki, Algebraic equivalence of locally normal

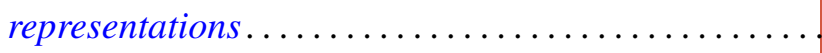

Joseph Earl Valentine, An analogue of Ptolemy's theorem and its converse in

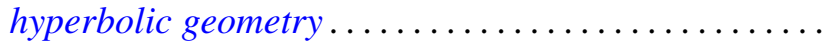

David Lawrence Winter, Solvability of certain p-solvable linear groups of finite order 